\title{
Zoonotic Fecal Pathogens and Antimicrobial Resistance in Canadian Petting Zoos
}

\author{
Cheyenne C. Conrad ${ }^{1,2}$ (1) ${ }^{\text {, Kim Stanford }}{ }^{1}$ (i) , Claudia Narvaez-Bravo ${ }^{3}$, Norman F. Neumann ${ }^{4}$, \\ Krysty Munns ${ }^{5}$, Lisa Tymensen ${ }^{1}$, Cassandra Jokinen ${ }^{1}$ and Tim A. McAllister ${ }^{5, *}$ \\ 1 Alberta Agriculture and Forestry, Lethbridge, AB T1J 4V6, Canada; cheyenne.conrad@agr.gc.ca (C.C.C.); \\ kim.stanford@gov.ab.ca (K.S.); lisa.tymensen@gov.ab.ca (L.T.); cassandra.jokinen@gov.ab.ca (C.J.) \\ 2 Canadian Association of Fairs and Exhibitions, Brandon, MB R7B 3W8, Canada \\ 3 Department of Food Science, University of Manitoba, Winnipeg, MB R3T 2N2, Canada; \\ Claudia.NarvaezBravo@umanitoba.ca \\ 4 Department of Public Health, University of Alberta, Edmonton, AB T6G 2G7, Canada; \\ nfneuman@ualberta.ca \\ 5 Agriculture and Agri-Food Canada, Lethbridge Research and Development Centre, Lethbridge, AB T1J 4B1, \\ Canada; krysty.munns@agr.gc.ca \\ * Correspondence: tim.mcallister@agr.gc.ca; Tel.: +1-403-317-2240
}

Received: 12 June 2018; Accepted: 11 July 2018; Published: 16 July 2018

\begin{abstract}
This study aimed to better understand the potential public health risk associated with zoonotic pathogens in agricultural fairs and petting zoos in Canada. Prevalence of Salmonella, Shiga toxin-producing Escherichia coli (STEC) O157:H7, and top six non-O157 STEC serogroups in feces ( $n=88)$, hide/feather $(n=36)$, and hand rail samples $(n=46)$ was assessed, as well as distributions of antimicrobial resistant (AMR) broad and extended-spectrum $\beta$-lactamase (ESBL)-producing $E$. coli. Prevalence of methicillin-resistant Staphylococcus aureus (MRSA) in pig nasal swabs $(n=4)$, and Campylobacter, Cryptosporidium, and Giardia in feces was also assessed. Neither Salmonella nor MRSA were detected. Campylobacter spp. were isolated from $32 \%$ of fecal samples. Cryptosporidium and Giardia were detected in $2 \%$ and $15 \%$ of fecal samples, respectively. Only one fecal sample was positive for STEC O157, whereas 22\% were positive for non-O157 STEC. Multi-drug resistance (MDR) to antibiotics classified as critically and highly important in human medicine was proportionally greatest in E. coli from cattle feces. The $\beta$-lactamase-producing E. coli from pig, horse/donkey feces, and hand rail samples, as well as the STEC E. coli from handrail swabs were MDR. The diversity and prevalence of zoonotic pathogens and AMR bacteria detected within agricultural fairs and petting zoos emphasize the importance of hygienic practices and sanitization with respect to reducing associated zoonotic risks.
\end{abstract}

Keywords: petting zoo; zoonoses; pathogens; Shiga toxin-producing Escherichia coli (STEC); methicillin-resistant Staphylococcus aureus (MRSA); antimicrobial resistance

\section{Introduction}

Agricultural fairs and petting zoos provide the public with the opportunity to interact and learn about farm animals. However, farm animals also present a risk for transmission of zoonotic pathogens [1]. Illnesses and outbreaks involving Shiga toxin-producing Escherichia coli (STEC) O157:H7 and other non-O157 serogroups, Campylobacter spp., Salmonella spp., Cryptosporidium spp., and to a lesser degree, Listeria monocytogenes and Yersinia enterocolitica have been reported in fair and petting zoo visitors [2,3]. It has been estimated that $14 \%$ of all illnesses caused by these pathogens arises from direct animal contact [3]. 
Humans can become infected with zoonotic pathogens by hand-to-mouth ingestion of animal feces or through direct contact with animals or contaminated surfaces [2,4]. The severity of illness can vary, but often results in self-limiting episodes of gastroenteritis characterized by abdominal cramps, nausea, vomiting, fever, and watery or bloody diarrhea which occasionally becomes hemorrhagic $[5,6]$. Severe cases can cause kidney damage or even death [5]. Studies have shown that petting zoo visitors frequently engage in practices that promote the transmission of pathogens such as touching their face after animal contact, letting animals lick their hands, or eating and drinking within animal enclosures [7]. Furthermore, several studies have found that hand-washing compliance by visitors is often poor ranging from $0 \%$ to $77 \%[1,7,8]$.

An additional concern for both human and animal health is the transmission of antimicrobial resistant (AMR) bacteria [9]. The emergence of bacteria resistant to "critically important" (e.g., third and fourth generation cephalosporins, streptomycin, ampicillin) or 'highly important' (e.g., sulfamethoxazole, trimethoprim, chloramphenicol, tetracyclines) antibiotics is of particular concern, as there are limited therapeutic options to treat infections caused by these bacteria [9-11]. Although generic E. coli are typically not pathogenic, they may harbor antibiotic resistance and virulence genes that can be transferred to other bacteria, including pathogens.

To better understand potential public health risks associated with petting zoos, the presence of the most prominent zoonotic pathogens associated with animal feces (i.e., STEC O157:H7, the top 6 non-O157 serogroups [12] O26, O45, O103, O111, O121, and O145, Campylobacter, Cryptosporidium, Giardia, and Salmonella), broad- and extended-spectrum $\beta$-lactamase (ESBL) resistant E. coli, as well as methicillin-resistant Staphylococcus aureus (MRSA) as indicator species for antibiotic resistance, were assessed in samples collected from animals and their environment at fairs and petting zoos in Canada. Though guidelines exist for maintaining hygienic and safe animal exhibits to reduce the risk of the public ingesting pathogens, there are no official regulations or inspections to enforce these measures. An online survey was distributed to livestock fair exhibitors across Canada to gain a broader understanding of the hygienic practices and safety precautions employed at these events.

\section{Materials and Methods}

\subsection{Survey Data Collection}

An online questionnaire was distributed to managers of 782 fairs and animal exhibitions across Canada (Enigma Research Corporation, Toronto, ON Canada). The survey included a variety of questions regarding the characteristics and cleaning practices of livestock pavilions and petting zoos at fairs.

\subsection{Sample Collection and Preparation}

Over two consecutive years, hand rail swabs $(n=46)$, hide/feather swabs $(n=36)$, composite fecal samples $(n=88)$, and nasal swabs $(n=4)$ were collected from a permanent seasonal petting zoo and mobile petting zoos located in Alberta $(n=5)$ and Manitoba $(n=1)$. Samples were processed according to Figure 1. Hand rails (approximately $75 \mathrm{~cm}^{2}$ ) and animal hides/feathers (neck and back areas, $100 \mathrm{~cm}^{2}$ ) were swabbed with sterile, moistened sponges (3M, St. Paul, MN, USA) and placed in buffered peptone water (BPW; Hardy Diagnostics, Santa Maria, CA, USA). Fresh feces from cows, calves, donkeys, horses, sheep, goats, llamas, pigs, and birds (i.e., ducks, chickens, parakeets, and peacocks) were collected from pen floors, pooled ( 5 samples from different animals of the same species), and homogenized. A sub-sample was added to Cary-Blair medium (Dalynn Biologicals, Calgary, AB, Canada). Nasal swabs (BBL CultureSwab, Cary-Blair transport medium, BD Canada, Mississauga, $\mathrm{ON}$, Canada) were only collected from pigs. Samples were maintained at $4{ }^{\circ} \mathrm{C}$ until processing.

In the laboratory, feces were frozen for Cryptosporidium and Giardia analysis. Sponge swabs in BPW were pre-enriched at $37^{\circ} \mathrm{C}$ overnight $(\sim 18 \mathrm{~h})$ for Salmonella, STEC, generic E. coli, and ESBL-producing 
E. coli. Feces from composite samples were homogenized in BPW for 1 min at 230 RPM using a Seward Model 400 stomacher (Cole-Parmer Canada, Montreal, QC, Canada).
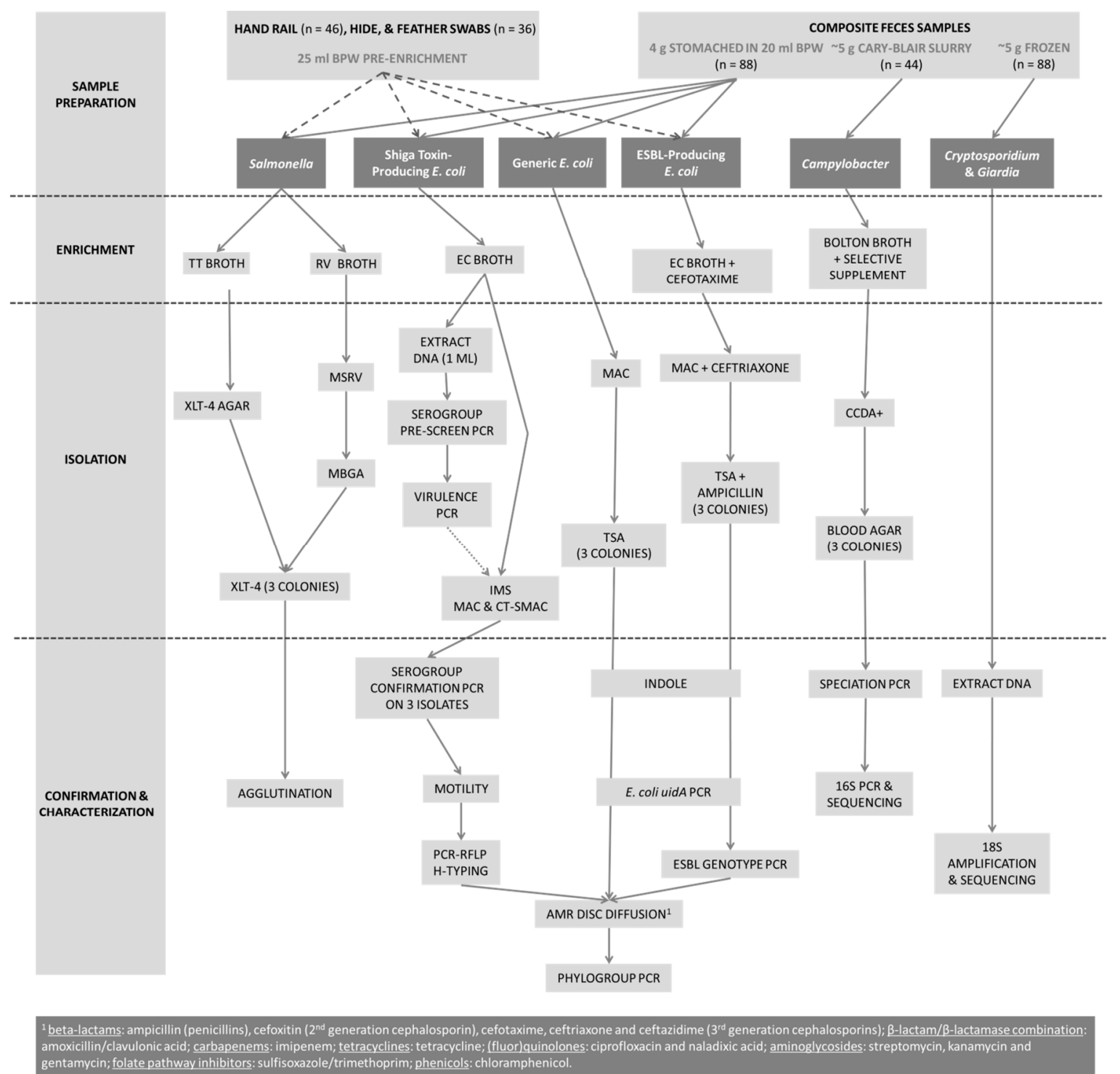

Figure 1. Flow chart representation of study methodology.

\subsection{Salmonella Screening}

Alongside positive (Salmonella Typhimurium ATCC 14028) and negative (E. coli ATCC 25922) controls, feces and sponge swab washings were each dispensed into Tetrathionate (TT; BD Canada, Mississauga, ON, Canada) and Rappaport-Vassiliadis (RV; EMD Millipore, Etobicoke, ON, Canada) broths and incubated at $42{ }^{\circ} \mathrm{C}$ for $20-24 \mathrm{~h}$. Tetrathionate enrichments were streaked onto Xylose Lysine Tergitol 4 agar (XLT-4; BD Canada, Mississauga, ON, Canada) and RV enrichments were transferred to modified semisolid RV (MSRV) agar plates and incubated at $37^{\circ} \mathrm{C}$ for $24 \mathrm{~h}$. A loop of MSRV culture from the edge of opaque zones was streaked onto modified Brilliant Green agar (MBGA; Hardy Diagnostics, Santa Maria, CA, USA) and incubated overnight at $37^{\circ} \mathrm{C}$ for $24 \mathrm{~h}$. Up to three colonies per XLT-4 and MBGA plate were sub-cultured to fresh XLT-4 plates and incubated at $37^{\circ} \mathrm{C}$ for $24 \mathrm{~h}$. Presumptive Salmonella isolates were tested for agglutination with Poly A-I + Vi antiserum (BD Canada, Mississauga, ON, Canada). 


\subsection{STEC Screening}

Feces and sponge swab washings were enriched for O157 and the top 6 non-O157 STEC in EC broth (EMD Millipore Etobicoke, ON, Canada) at $37^{\circ} \mathrm{C}$ for $6 \mathrm{~h}$. Cultures were centrifuged $(10 \mathrm{~min}$ at $6000 \times g$ ) and DNA was extracted using a NucleoSpin Tissue kit (Macherey-Nagel, Düren, Germany) according to manufacturer's instructions.

Targeted immunomagnetic separation (IMS; Romer Labs, Newark, DE, USA) with plating onto MacConkey (MAC) agar and sorbitol MAC agar containing Cefixime Tellurite (CT-SMAC) was conducted on PCR-positive cultures for retrieval of non-O157 and O157 cells, respectively [13]. Serogroup confirmation and the presence of st $x 1$, st $x 2$, eae, and $e h x A$ virulence genes were determined by PCR [14]. Isolates were screened for motility (Hardy Diagnostics, Santa Maria, CA, USA), followed by flagellar (H-antigen) typing as previously described [15].

\subsection{Generic E. coli Screening}

Feces and pre-enriched swabs were streaked onto MAC agar and incubated overnight at $37^{\circ} \mathrm{C}$. Presumptive E. coli was streaked onto Tryptic Soy Agar (TSA) and incubated overnight at $37^{\circ} \mathrm{C}$, followed by testing for indole production (Ricca Chemical Co., Arlington, TX, USA). Lactose-fermenting and indole-producing isolates were screened via PCR for the presence of the E. coli-specific uidA gene $[16,17]$.

\subsection{Broad-and Extended-Spectrum $\beta$-Lactamase E. coli Screening}

Feces and swab washings were added to EC broth containing cefotaxime $(2 \mu \mathrm{g} / \mathrm{mL})$ and incubated overnight at $37^{\circ} \mathrm{C}$. Enrichments were streaked onto MAC plates containing ceftriaxone $(1 \mu \mathrm{g} / \mathrm{mL})$ and incubated overnight at $37^{\circ} \mathrm{C}$. Typical E. coli colonies were streaked onto TSA containing ampicillin (32 $\mathrm{\mu g} / \mathrm{mL}$ ) and incubated overnight at $37^{\circ} \mathrm{C}$. Indole tests were completed as described above.

Broad- and extended-spectrum $\beta$-lactamase E. coli genotypes were determined by PCR for

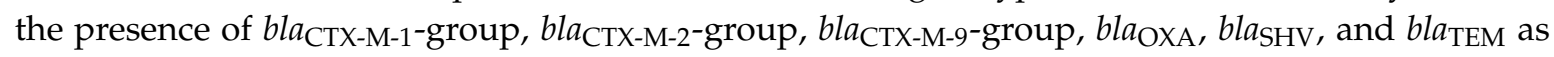
described previously [18]. PCR products were purified (Qiagen, Toronto, ON, Canada), sequenced (Eurofins Genomics, Louisville, KY, USA), and compared to ESBL-gene sequences within GenBank using BLAST.

\subsection{Campylobacter Screening}

In the second year, all fecal samples were screened for Campylobacter along with positive (Campylobacter coli ATCC 33559, Campylobacter jejuni ATCC 49943) and negative (Escherichia coli ATCC 25922) controls. Fecal slurries were added to Bolton broth containing selective supplement (BB+; Oxoid, Nepean, ON, Canada) and 5\% laked horse blood (Oxoid, Nepean, ON, Canada). Enrichments were placed into an AnaeroJar with 2.5 L CampyGen sachets (Oxoid, Nepean, ON, Canada) to maintain microaerophilic conditions $\left(5 \% \mathrm{O} 2,10 \% \mathrm{CO}_{2}\right)$, and incubated for $2 \mathrm{~h}$ at $37^{\circ} \mathrm{C}$, followed by $20-22 \mathrm{~h}$ at $41.5^{\circ} \mathrm{C}$. As described previously [19], $100 \mu \mathrm{L}$ of enriched BB+ culture was expelled onto a mixed cellulose ester membrane (0.65 $\mu \mathrm{m}$; Millipore Ltd., Etobicoke, ON, Canada) placed on the surface of blood-free charcoal cefoperazone deoxycholate agar (CCDA+; Oxoid, Nepean, ON, Canada) containing selective supplement. After $15 \pm 0.5 \mathrm{~min}$, membranes were removed and discarded and CCDA+ plates were incubated for $24-48 \mathrm{~h}$ at $42{ }^{\circ} \mathrm{C}$ under microaerophilic conditions. Presumptive campylobacters were sub-cultured to blood agar (BD Canada, Mississauga, ON, Canada) containing 5\% defibrinated sheep blood (Oxoid, Nepean, ON, Canada). To determine species (C. coli or C. jejuni), PCR assays were prepared using HotStar master mix (Qiagen, Toronto, ON, Canada) according to manufacturer's instructions with a final primer concentration of $1000 \mathrm{nM}$ [20]. C. coli and/or C. jejuni PCR-negative isolates were subjected to $16 \mathrm{~S}$ rRNA PCR using primers $954 \mathrm{~F}$ and $1369 \mathrm{R}[21,22]$ at a final concentration of $200 \mathrm{nM}$ and sequenced (Eurofins Genomics, Toronto, ON, Canada). 


\subsection{Cryptosporidium and Giardia Screening}

Cryptosporidium oocysts were extracted from fecal samples using the PowerFecal ${ }^{\circledR}$ extraction kit (Qiagen, Toronto, ON, Canada). Briefly, $0.25 \mathrm{~g}$ of feces were suspended in buffers $\mathrm{C} 1$ and $\mathrm{C} 2$, followed by five freeze-thaw cycles, alternating between a dry ice/ethanol bath and $95{ }^{\circ} \mathrm{C}$ heating block. The remainder of the extraction followed manufacturer's instructions. Cryptosporidium spp. were detected in the extracts by amplification of $18 \mathrm{~S}$ rRNA as previously described [23] using Maxima Hot Start PCR Master Mix (Thermo Fisher Scientific, Toronto, ON, Canada) and final primer concentrations of $500 \mathrm{nM}$. Reactions were visualized on 1\% agarose gels and $18 \mathrm{~S}$ bands were extracted with the QIAquick gel extraction kit (Qiagen, Toronto, ON, Canada). Sanger sequencing was performed on $18 \mathrm{~S}$ bands (Macrogen, Seoul, Korea). Genotype/species of Cryptosporidium-positive samples were identified based on the bioinformatic methods of Ruecker et al. [24].

A $292 \mathrm{bp}$ fragment of the 16S small-subunit ribosomal RNA (SSU rRNA) was used to detect Giardia intestinalis and identification of genetic assemblages using endpoint PCR as described by Hopkins et al. [25] along with modifications as described by Smith et al. [26].

\subsection{MRSA Screening}

Alongside positive (S. aureus ATCC 43300) and negative (S. aureus ATCC 49775) controls, nasal swabs were transferred to SA broth $(10 \mathrm{~g}$ tryptone/L, $75 \mathrm{~g}$ sodium chloride/L, $10 \mathrm{~g}$ mannitol/L and $2.5 \mathrm{~g}$ of yeast extract/L) and incubated at $35^{\circ} \mathrm{C}$ for $24 \mathrm{~h}$, then streaked onto MRSA CHROMagar plates (Dalynn Biologicals, Calgary, AB, Canada) and incubated at $35^{\circ} \mathrm{C}$ for $24 \mathrm{~h}$. Presumptive S. aureus colonies were transferred to $300 \mu \mathrm{L}$ TSB and incubated at $35^{\circ} \mathrm{C}$ for $24 \mathrm{~h}$. Cultures were streaked onto MRSA CHROMagar prior to confirmation using the StaphTEX Blue latex agglutination test (Hardy Diagnostics, Santa Maria, CA, USA).

\subsection{Antimicrobial Resistance Testing}

A total of 583 E. coli isolates (425 generic, 99 putative-STEC, 59 ESBL-producing) and a control strain (E. coli ATCC 25922) were streaked onto TSA plates and incubated overnight at $37^{\circ} \mathrm{C}$. The direct colony method was used to prepare isolates for disk-diffusion testing according to CLSI M02-A12 [27]. The antibiotic panel (Sensi-disks, BD Canada) consisted of one or two representatives from eight classes (Figure 1). Disk-diffusion plate readings were completed using the BIOMIC $\mathrm{V}^{3}$ software version 7.1.1.2014 (Giles Scientific, Santa Barbara, CA, USA). Resistance was defined using established standards [28]. Multi-drug resistance (MDR) was defined as resistance $\geq 3$ different classes of antibiotics [29].

\subsection{E. coli Isolate Phylogroups}

Phylogroup determination based on PCR results was completed for all E. coli isolates as described by Clermont et al. [30]. Briefly, an E. coli isolate was assigned to one of 8 phylogroups based on presence or absence of the genes $\operatorname{arpA}$, chuA, yjaA, and TspE4.C2.

\subsection{Statistical Analysis}

Prevalence of STEC or Campylobacter spp. among sample types collected and animal species were compared with generalized linear mixed models (Proc Glimmix, SAS 9.3, SAS Institute Inc., Ottawa, ON, Canada) using a binomial distribution. Model adjusted means (back-transformed to original scale) were reported and deemed significant at $p<0.05$. 


\section{Results}

\subsection{Survey}

Survey responses are summarized in Table 1 . The survey had a completion rate of $24 \%$ (187 completed questionnaires). Visitors were allowed food within the animal contact area at $51 \%$ of petting zoos and $71 \%$ of livestock pavilions at fairs. Reportedly, 31\% and $23 \%$ of livestock pavilions and petting zoos, respectively, never sanitize any surfaces. Furthermore, nearly $30 \%$ of petting zoos and livestock pavilions did not provide hand-washing facilities or hand sanitizer. Only $2 \%$ of fairs reported an outbreak within the last 10 years; with the majority $(75 \%)$ identifying E. coli as the causative agent.

\subsection{Prevalence of 0157 and Non-O157 STEC, Salmonella, Campylobacter, MRSA, Cryptosporidium and} Giardia.

STEC O157 isolation frequency was lowest overall, isolated only from feces ( $0.6 \%$ of samples), while non-O157 STEC were isolated from all sources (22\%), including hides / feathers and wooden hand rails (Table 2). Neither Salmonella nor MRSA were isolated from any samples. Cryptosporidium and Giardia were detected in $2 \%$ and $15 \%$ of fecal samples, respectively. Campylobacter spp. were the most prevalent and were isolated from $30 \%$ of fecal samples.

\subsection{Screening for STEC and Related Genes}

A viable putative-STEC isolate was obtained from $23 \%$ of samples. Following PCR detection of st $x 1$ and st $x 2$ toxin genes, 66 isolates were confirmed to be STEC. Serogroups O26 and O103 were observed at most petting zoos and were the most frequent overall (Table 3). Resistance to antibiotics, albeit to only one class, was most common for $\mathrm{O} 26$ isolates. Resistance was more likely for isolates of serogroup $\mathrm{O} 26$ than $\mathrm{O} 103(p<0.05)$. The $\mathrm{O} 26$ isolates showing resistance to kanamycin and streptomycin were primarily isolated from petting zoo A, while $\mathrm{O} 26$ isolates resistant to tetracycline originated primarily from petting zoo F. Three MDR serotypes (O45:H21, O45:H19, O103:H2) from either feather or hand rail swabs, were also collected from petting zoos A and F. All O26 and O45 isolates contained stx1, while other virulence markers were variable across serogroups. All O145 isolates contained eae and st $x 1$ regardless of sample type or petting zoo. All O103:H38 isolates possessed ehxA, eae, and st 1 genes regardless of host, sample type, phylogenetic group, or petting zoo. 
Table 1. Summary of the survey results concerning livestock pavilions and petting zoos at fairs across Canada.

\begin{tabular}{|c|c|c|c|}
\hline Question & Answer & & airs \\
\hline \multirow{4}{*}{ How many visitors attend the fair each year? $(n=185)$} & under 5000 visitors & 36 & \\
\hline & $5000-19,999$ visitors & 36 & \\
\hline & $20,000-99,999$ visitors & 20 & \\
\hline & 100,000 and over & 8 & \\
\hline \multirow{6}{*}{ Where is the fair located? $(n=185)$} & Alberta & 14 & \\
\hline & British Columbia & 11 & \\
\hline & Manitoba & 5 & \\
\hline & Ontario & 49 & \\
\hline & Quebec & 9 & \\
\hline & Other & 12 & \\
\hline \multirow{2}{*}{$\begin{array}{l}\text { Is there a petting zoo featured at the fair? }(n=171) \\
\text { Is there a livestock pavilion or barn? }(n=184)\end{array}$} & Yes & 71 & \\
\hline & Yes & 72 & \\
\hline $\begin{array}{l}\text { Has there been an outbreak or problem with a pathogen in the past } \\
10 \text { years? }(n=164)\end{array}$ & Yes & 2 & \\
\hline \multirow{2}{*}{$\begin{array}{l}\text { What was the pathogen of concern? (among those that reported a } \\
\text { problem or outbreak) }(n=4)\end{array}$} & E. coli & 75 & \\
\hline & Unknown & 25 & \\
\hline Question & Answer & \% Livestock Pavilions at fairs & $\%$ Petting Zoos at fairs \\
\hline \multirow{9}{*}{ Which animal species are present at the facility? $(\mathrm{L} n=131 ; \mathrm{P} n=119)$} & Cattle and Calves & 89 & 44 \\
\hline & Horses and Ponies & 77 & 71 \\
\hline & Sheep & 69 & 72 \\
\hline & Goats & 55 & 78 \\
\hline & Rabbits & 62 & 73 \\
\hline & Pigs & 40 & 52 \\
\hline & Donkeys & NA & 52 \\
\hline & Alpacas or Llamas & NA & 36 \\
\hline & Guinea Pigs & NA & 19 \\
\hline \multirow{4}{*}{$\begin{array}{l}\text { What is the level of contact between humans and animals within } \\
\text { petting zoos? }(n=117)\end{array}$} & $\begin{array}{l}\text { Animals roam freely without barriers and visitors can pet, } \\
\text { hold, and feed the animals }\end{array}$ & NA & 17 \\
\hline & $\begin{array}{l}\text { Animals roam freely without barriers visitors can pet and } \\
\text { hold the animals }\end{array}$ & NA & 5 \\
\hline & $\begin{array}{l}\text { A barrier separates animals and visitors, and visitors can } \\
\text { pet and feed the animals }\end{array}$ & NA & 44 \\
\hline & $\begin{array}{l}\text { A barrier separates animals and visitors, and visitors can } \\
\text { only pet the animals }\end{array}$ & NA & 34 \\
\hline \multirow{3}{*}{$\begin{array}{l}\text { What is the level of contact between humans and animals at the } \\
\text { livestock pavilion? }(n=131)\end{array}$} & None, only exhibitors have contact with the animals & 11 & NA \\
\hline & Public is permitted to walk through the livestock area & 71 & NA \\
\hline & Public is encouraged to touch or interact with the animals & 18 & NA \\
\hline
\end{tabular}


Table 1. Cont.

\begin{tabular}{|c|c|c|c|}
\hline Question & Answer & & airs \\
\hline Is there a children's play area directly adjacent? $(\mathrm{L} n=130 ; \mathrm{P} n=115)$ & Yes & 18 & 30 \\
\hline $\begin{array}{l}\text { Is there a food or beverage area directly adjacent? }(\mathrm{L} n=131 ; \\
\mathrm{P} n=116)\end{array}$ & Yes & 16 & 2 \\
\hline $\begin{array}{l}\text { Are there rules regarding visitors bringing food or drink into the } \\
\text { animal area? }(\mathrm{L} n=125 ; \mathrm{P} n=117)\end{array}$ & $\begin{array}{l}\text { Not allowed } \\
\text { Allowed, but no touching animals } \\
\text { Allowed, not for feeding animals } \\
\text { No restrictions }\end{array}$ & $\begin{array}{l}29 \\
7 \\
25 \\
39\end{array}$ & $\begin{array}{l}49 \\
5 \\
25 \\
21\end{array}$ \\
\hline Is there continuous removal of feces? $(\mathrm{L} n=103 ; \mathrm{P} n=84)$ & Yes & 78 & 69 \\
\hline $\begin{array}{l}\text { What is the frequency of removal of feces from the animal area? } \\
(\mathrm{L} n=117 ; \mathrm{P} n=111)\end{array}$ & $\begin{array}{l}\text { Immediately } \\
2-4 \text { times daily } \\
\text { Once daily } \\
\text { Less frequently }\end{array}$ & $\begin{array}{l}57 \\
32 \\
8 \\
3\end{array}$ & $\begin{array}{l}60 \\
32 \\
8 \\
0\end{array}$ \\
\hline Are there hand-washing stations? ( $\mathrm{L} n=123 ; \mathrm{P} n=117)$ & Yes & 73 & 69 \\
\hline Are there hand sanitizers? $(\mathrm{L} n=96 ; \mathrm{P} n=96)$ & Yes & 73 & 79 \\
\hline $\begin{array}{l}\text { Does the staff remind visitors to wash their hands? ( } \mathrm{L} n=117 \text {; } \\
\mathrm{P} n=111 \text { ) }\end{array}$ & Yes & 44 & 69 \\
\hline Is there hand-washing signage? $(\mathrm{L} n=97 ; \mathrm{P} n=88)$ & Yes & 73 & 72 \\
\hline Is there no smoking signage? ( $\mathrm{L} n=89 ; \mathrm{P} n=63)$ & Yes & 67 & 52 \\
\hline Is there no eating signage? ( $\mathrm{L} n=39 ; \mathrm{P} n=43)$ & Yes & 30 & 35 \\
\hline How often are surfaces sanitized? ( $\mathrm{L} n=111 ; \mathrm{P} n=107)$ & $\begin{array}{l}\text { Multiple times a day } \\
\text { Once daily } \\
\text { Only when visibly dirty } \\
\text { Never }\end{array}$ & $\begin{array}{l}18 \\
19 \\
32 \\
31 \\
\end{array}$ & $\begin{array}{l}30 \\
20 \\
27 \\
23\end{array}$ \\
\hline Is there a safe area for storage of visitor items? ( $\mathrm{L} n=117 ; \mathrm{P} n=113)$ & Yes & 25 & 30 \\
\hline
\end{tabular}

$\mathrm{NA}=$ not applicable. $\mathrm{L}=$ Livestock Pavilions. $\mathrm{P}=$ Petting Zoos 
Table 2. Prevalence of O157 and the top 6 non-O157 STEC, Salmonella, Campylobacter spp., Cryptosporidium and Giardia from samples collected at petting zoos in Canada (2015-2016).

\begin{tabular}{|c|c|c|c|c|c|c|c|c|c|}
\hline \multirow{2}{*}{ Sample Type } & \multirow{2}{*}{ Host } & \multicolumn{2}{|c|}{$\%$ STEC } & \multirow{2}{*}{$\%$ Salmonella } & \multicolumn{3}{|c|}{$\%$ Campylobacter 1} & \multirow{2}{*}{$\%$ Cryptosporidium } & \multirow{2}{*}{$\%$ Giardia $^{6}$} \\
\hline & & O157 & Non-O157 & & jejuni & coli & lanienae & & \\
\hline \multirow{6}{*}{$\begin{array}{c}\text { Composite } \\
\text { Fecal Samples }\end{array}$} & Cattle & $0(0 / 9)$ & $44(4 / 9)$ & $0(0 / 9)$ & $0(0 / 5)$ & $0(0 / 5)$ & $0(0 / 5)$ & $0(0 / 9)$ & $22(2 / 9)$ \\
\hline & Sheep and Goats & $3(1 / 36)$ & $19(7 / 36)$ & $0(0 / 36)$ & $7(1 / 15)$ & $7(1 / 15)$ & $0(0 / 15)$ & $6(2 / 36)^{5}$ & $25(9 / 36)$ \\
\hline & Pigs & $0(0 / 12)$ & $33(4 / 12)$ & $0(0 / 12)$ & $0(0 / 10)$ & $20(2 / 10)$ & $30(3 / 10)$ & $0(0 / 12)$ & $0(0 / 12)$ \\
\hline & Horses and Donkeys & $0(0 / 18)$ & $28(5 / 18)$ & $0(0 / 18)$ & $0(0 / 8)$ & $38(3 / 8)^{2}$ & $25(2 / 8)^{2}$ & $0(0 / 18)$ & $11(2 / 18)$ \\
\hline & Llamas & $0(0 / 6)$ & $100(6 / 6)$ & $0(0 / 6)$ & $\mathrm{NT}^{4}$ & $\mathrm{NT}^{4}$ & $\mathrm{NT}^{4}$ & $0(0 / 6)$ & $0(0 / 6)$ \\
\hline & Birds $^{3}$ & $0(0 / 7)$ & $29(2 / 7)$ & $0(0 / 7)$ & $17(1 / 6)$ & $17(1 / 6)$ & $0(0 / 6)$ & $0(0 / 7)$ & $0(0 / 7)$ \\
\hline \multirow[t]{2}{*}{ Total } & & $1(1 / 88)$ & $32(28 / 88)$ & $0(0 / 88)$ & $5(2 / 44)$ & $16(7 / 44)$ & $11(5 / 44)$ & $2(2 / 88)$ & $15(13 / 88)$ \\
\hline & Cattle & $0(0 / 4)$ & $0(0 / 4)$ & $0(0 / 4)$ & $\mathrm{NT}^{4}$ & $\mathrm{NT}^{4}$ & $\mathrm{NT}^{4}$ & $\mathrm{NT}^{4}$ & $\mathrm{NT}^{4}$ \\
\hline \multirow{6}{*}{$\begin{array}{c}\text { Hide/Feather } \\
\text { Swabs }\end{array}$} & Sheep and Goats & $0(0 / 15)$ & $7(1 / 15)$ & $0(0 / 15)$ & $\mathrm{NT}^{4}$ & $\mathrm{NT}^{4}$ & $\mathrm{NT}^{4}$ & $\mathrm{NT}^{4}$ & $\mathrm{NT}^{4}$ \\
\hline & Pigs & $0(0 / 5)$ & $20(1 / 5)$ & $0(0 / 5)$ & NT 4 & $\mathrm{NT}^{4}$ & NT 4 & NT 4 & $\mathrm{NT}^{4}$ \\
\hline & Horses and Donkeys & $0(0 / 5)$ & $0(0 / 5)$ & $0(0 / 5)$ & NT 4 & $\mathrm{NT}^{4}$ & $\mathrm{NT}^{4}$ & NT 4 & $\mathrm{NT}^{4}$ \\
\hline & Llamas & $0(0 / 3)$ & $0(0 / 3)$ & $0(0 / 3)$ & NT 4 & $\mathrm{NT}^{4}$ & NT 4 & $\mathrm{NT}^{4}$ & NT 4 \\
\hline & Birds $^{3}$ & $0(0 / 3)$ & $100(3 / 3)$ & $0(0 / 3)$ & $\mathrm{NT}^{4}$ & $\mathrm{NT}^{4}$ & $\mathrm{NT}^{4}$ & $\mathrm{NT}^{4}$ & $\mathrm{NT}^{4}$ \\
\hline & Rabbits & $0(0 / 1)$ & $0(0 / 1)$ & $0(0 / 1)$ & $\mathrm{NT}^{4}$ & $\mathrm{NT}^{4}$ & $\mathrm{NT}^{4}$ & $\mathrm{NT}^{4}$ & $\mathrm{NT}^{4}$ \\
\hline Total & & $0(0 / 36)$ & $14(5 / 36)$ & $0(0 / 36)$ & & & & & \\
\hline Hand Rail & Wood & $0(0 / 28)$ & $14(4 / 28)$ & $0(0 / 28)$ & $\mathrm{NT}^{4}$ & $\mathrm{NT}^{4}$ & $\mathrm{NT}^{4}$ & $\mathrm{NT}^{4}$ & $\mathrm{NT}^{4}$ \\
\hline Swabs & Metal & $0(0 / 18)$ & $0(0 / 18)$ & $0(0 / 18)$ & NT 4 & NT 4 & $\mathrm{NT}^{4}$ & $\mathrm{NT}^{4}$ & $\mathrm{NT}^{4}$ \\
\hline Total & & $0(0 / 46)$ & $9(4 / 46)$ & $0(0 / 46)$ & & & & & \\
\hline Grand Total & & $0.6(1 / 170)$ & $22(37 / 170)$ & $0(0 / 170)$ & $5(2 / 44)$ & $16(7 / 44)$ & $11(5 / 44)$ & $2(2 / 88)^{5}$ & $15(13 / 88)^{6}$ \\
\hline
\end{tabular}

Birds $=$ Chickens, ducks, peacocks, turkeys, parakeets. ${ }^{4} \mathrm{NT}=$ Not tested. ${ }^{5} 1 \mathrm{C}$. bovis, and $1 \mathrm{C}$. xiaoi. ${ }^{6}$ All G. intestinalis assemblage A. 
Table 3. Characteristics of STEC isolates $(n=66)$

\begin{tabular}{|c|c|c|c|c|c|c|c|c|c|}
\hline Serogroup & H-Type & Motility $^{1}$ & $\begin{array}{l}\text { Petting Zoo } \\
\text { ID }^{2}\end{array}$ & Sample Type & Host & Virulence Markers & $\begin{array}{l}\text { Phylogenetic } \\
\text { Group }^{3}\end{array}$ & \# of Isolates & $\begin{array}{c}\text { Antibiotic Resistance Pattern (\# } \\
\text { Antibiotic Classes) }{ }^{4,5}\end{array}$ \\
\hline $\mathrm{O} 26$ & $\mathrm{H} 34$ & + & A2 & feces & cattle & ehxA, eae, stx1 & $\mathrm{U}$ & 1 & NA \\
\hline $\mathrm{O} 26$ & $\mathrm{H} 46$ & + & A2 & feces & cattle & stx1 & $\mathrm{U}$ & 1 & KAN-STREP (1) \\
\hline $\mathrm{O} 26$ & $\mathrm{H} 46$ & + & A2 & feces & cattle & ehxA, stx1 & U & 1 & KAN-STREP (1) \\
\hline $\mathrm{O} 26$ & $\mathrm{H} 34$ & + & A2 & feces & cattle & stx1 & Multiple ${ }^{6}$ & 2 & NA \\
\hline $\mathrm{O} 26$ & $\mathrm{H} 46$ & + & $\mathrm{F}$ & feces & donkey & ehxA, eae, stx1, stx2 & A & 1 & TET (1) \\
\hline $\mathrm{O} 26$ & $\mathrm{H} 46$ & + & A1 & feces & donkey & eae, stx1 & $\mathrm{U}$ & 1 & KAN-STREP (1) \\
\hline $\mathrm{O} 26$ & $\mathrm{H} 46$ & + & A1 & feces & donkey & ehxA, stx1 & $\mathrm{U}$ & 1 & KAN-STREP (1) \\
\hline $\mathrm{O} 26$ & $\mathrm{H} 46$ & + & $\mathrm{A} 1, \mathrm{~F}$ & feces & donkey & stx1 & $\mathrm{U}$ & $4^{7}$ & KAN-STREP (1) \\
\hline $\mathrm{O} 26$ & $\mathrm{H} 46$ & + & A2 & feces & donkey & stx1 & A & 2 & TET (1) \\
\hline $\mathrm{O} 26$ & H11 & + & $\mathrm{C} 1$ & feces & goat & ehxA, eae, stx1 & $\mathrm{U}$ & $2^{7}$ & NA \\
\hline $\mathrm{O} 26$ & $\mathrm{H} 46$ & + & A2 & feces & goat & eae, stx1 & $\mathrm{U}$ & 1 & KAN-STREP (1) \\
\hline $\mathrm{O} 26$ & $\mathrm{H} 46$ & + & $\mathrm{F}$ & feces & goat & stx1, stx2 & A & 1 & TET (1) \\
\hline $\mathrm{O} 26$ & $\mathrm{H} 46$ & + & $\mathrm{F}$ & feces & horse & eae, stx1, stx2 & A & 1 & TET (1) \\
\hline $\mathrm{O} 26$ & H34 & + & A2 & feces & llama & stx1 & B2 & $2^{7}$ & NA \\
\hline $\mathrm{O} 26$ & $\mathrm{H} 46$ & + & $\mathrm{F}$ & feces & pig & stx1, stx2 & $\mathrm{A}$ & $3^{7}$ & TET (1) \\
\hline $\mathrm{O} 26$ & $\mathrm{H} 46$ & + & $\mathrm{F}$ & feather swab & chicken & stx1 & A & 1 & TET (1) \\
\hline $\mathrm{O} 26$ & $\mathrm{H} 46$ & + & $\mathrm{F}$ & hand rail & wood & stx1 & A & 1 & NA \\
\hline $\mathrm{O} 45$ & H34 & + & A2 & feces & llama & eae, stx1 & $\mathrm{U}$ & 1 & NA \\
\hline $\mathrm{O} 45$ & $\mathrm{H} 21$ & NM & $\mathrm{F}$ & hand rail & wood & ehxA, eae, stx1 & $\mathrm{U}$ & 1 & CHL-STREP-TET-TMS (4) \\
\hline $\mathrm{O} 45$ & H19 & NM & $\mathrm{F}$ & feather swab & chicken & stx1 & $\mathrm{E}$ & 1 & $\begin{array}{l}\text { AMC-AMP-CAZ-CFX-CHL- } \\
\text { CRO-CTX-GEN-STREP-TET (5) }\end{array}$ \\
\hline O103 & $\mathrm{H} 43$ & + & B & feces & cattle & ehxA, eae, stx2 & B1 & 1 & NA \\
\hline O103 & $\mathrm{H} 43$ & + & B & feces & cattle & stx2 & $\mathrm{U}$ & 1 & NA \\
\hline O103 & $\mathrm{H} 43$ & + & B & feces & cattle & stx1 & B1 & 1 & NA \\
\hline $\mathrm{O} 103$ & $\mathrm{H} 38$ & + & $\mathrm{F}$ & feces & donkey & ehxA, eae, stx1 & B1 & 2 & NA \\
\hline O103 & $\mathrm{H} 38$ & + & $\mathrm{F}$ & feces & duck & ehxA, eae, stx1 & Multiple ${ }^{8}$ & $3^{7}$ & NA \\
\hline O103 & H38 & + & $\mathrm{F}$ & feces & goat & ehxA, eae, stx1 & B1 & 2 & NA \\
\hline O103 & $\mathrm{H} 43$ & + & B & feces & sheep or goat & stx2 & B1 & 1 & NA \\
\hline $\mathrm{O} 103$ & $\mathrm{H} 43$ & + & B & feces & sheep or goat & stx1 & $\mathrm{E}$ & 1 & NA \\
\hline O103 & H19 & + & B & feces & sheep or goat & stx2 & $\mathrm{U}$ & 1 & NA \\
\hline O103 & $\mathrm{H} 38$ & + & $\mathrm{F}$ & feces & horse & ehxA, eae, stx1 & B1 & 1 & NA \\
\hline O103 & $\mathrm{H} 21$ & + & $\mathrm{F}$ & feces & horse & stx1 & B1 & 1 & NA \\
\hline $\mathrm{O} 103$ & $\mathrm{H} 38$ & + & $\mathrm{F}$ & feces & pig & ehxA, eae, stx1 & B1 & 1 & NA \\
\hline O103 & H19 & + & $\mathrm{C} 2$ & feces & pig & stx2 & B1 & 1 & NA \\
\hline O103 & $\mathrm{H} 2$ & + & A2 & hand rail & wood & eae, stx1 & Multiple $^{9}$ & 3 & STREP-TET-TMS (3) \\
\hline O103 & $\mathrm{H} 4$ & + & $\mathrm{F}, \mathrm{C} 2$ & hand rail & wood & eae, st $x 1$ & B1 & $3^{7}$ & NA \\
\hline $\mathrm{O} 103$ & $\mathrm{H} 42$ & + & $\mathrm{C} 2$ & hand rail & wood & eae, stx1 & B1 & 1 & NA \\
\hline O103 & $\mathrm{H} 38$ & + & $\mathrm{F}$ & feather swab & duck & ehxA, eae, stx1 & B1 & 1 & NA \\
\hline O103 & H38 & + & $\mathrm{C} 1$ & hide swab & goat & ehxA, eae, stx1 & $\mathrm{E}$ & 1 & TET (1) \\
\hline
\end{tabular}


Table 3. Cont.

\begin{tabular}{|c|c|c|c|c|c|c|c|c|c|}
\hline Serogroup & H-Type & Motility $^{1}$ & $\begin{array}{l}\text { Petting Zoo } \\
\text { ID }^{2}\end{array}$ & Sample Type & Host & Virulence Markers & $\begin{array}{l}\text { Phylogenetic } \\
\text { Group }^{3}\end{array}$ & \# of Isolates & $\begin{array}{l}\text { Antibiotic Resistance Pattern (\# } \\
\text { Antibiotic Classes) } \\
4,5\end{array}$ \\
\hline O121 & H19 & + & A1 & feces & llama & eae, stx2 & B1 & 1 & NA \\
\hline O121 & $\mathrm{H7}$ & + & $\mathrm{C} 2$ & feces & pig & stx1 & B1 & 1 & TET (1) \\
\hline O145 & $\mathrm{H} 25$ & + & A1 & feces & llama & eae, stx1 & Multiple ${ }^{10}$ & $3^{7}$ & NA \\
\hline O145 & $\mathrm{H} 28$ & NM & $\mathrm{F}$ & feces & pig & eae, stx1 & E & 1 & NA \\
\hline $\mathrm{O} 145$ & $\mathrm{H} 25$ & + & E & hide swab & pig & eae, stx1 & B2 & 2 & NA \\
\hline O157 & H7 & + & $\mathrm{C} 1$ & feces & goat & ehxA, eae, stx1, stx2 & $\mathrm{E}$ & 3 & NA \\
\hline
\end{tabular}

${ }^{1}$ Motility test ( $+=$ motile; $\mathrm{NM}=$ non-motile). ${ }^{2}$ Petting zoos sampled: A1 ( $1^{\text {st }}$ sampling), A2 (2 ${ }^{\text {nd }}$ sampling), B, C1 ( $1^{\text {st }}$ sampling), C2 (2 ${ }^{\text {nd }}$ sampling), D, E, and F. ${ }^{3} \mathrm{U}=$ unknown.

${ }^{4} \mathrm{NA}=$ Not applicable. ${ }^{5} \mathrm{CHL}$, chloramphenicol; KAN, kanamycin; STREP, streptomycin; TET, tetracycline; TMS, trimethoprim/ sulfamethoxazole. ${ }^{6} 1$ isolate $=$ clade I; 1 isolate $=\mathrm{B} 2$.

7 Isolates collected from different samples. ${ }^{8} 1$ isolate B1, 2 isolates $=\mathrm{U} .{ }^{9} 1$ isolate $=\mathrm{U} ; 1$ isolate $=\mathrm{B} 1 ; 1$ isolate $=\mathrm{E}$. 1 isolate $=\mathrm{U} ; 2$ isolates $=\mathrm{B} 2$. 


\subsection{Screening for Broad-and Extended-Spectrum $\beta$-Lactamase E. coli}

Broad-spectrum $\beta$-lactamase (TEM-1) and ESBL (CTX-M) -producing E. coli $(n=59)$ were observed in $23.9 \%$ fecal samples. However, resistance determinants were only identified in $10.2 \%$ of these samples (21 isolates) (Table 4). TEM-1 was most common $(n=10)$, followed by CTX-M-15 $(n=8)$, and CTX-M-1 $(n=3)$.

Table 4. Distribution of phylogenetic subgroups among broad- and extended-spectrum $\beta$-lactamase $E$. coli genotypes isolated from the feces of petting zoo animals $(n=9)$.

\begin{tabular}{|c|c|c|c|}
\hline ESBL Genotype & Animal Group & Phylogenetic Group ${ }^{1}$ & \# Isolates \\
\hline blactX-M-1 & cattle & $\mathrm{U}$ & 3 \\
\hline \multirow{5}{*}{ bla } & birds & $\mathrm{A}$ & 1 \\
\hline & birds & B1 & 1 \\
\hline & birds & $\mathrm{U}$ & 1 \\
\hline & goats & B1 & 3 \\
\hline & pigs & B1 & 2 \\
\hline \multirow{5}{*}{ bla $_{\text {TEM-1 }}$} & cattle & B1 & 4 \\
\hline & cattle & $\mathrm{E}$ & 1 \\
\hline & cattle & $\mathrm{U}$ & 1 \\
\hline & donkeys & $\mathrm{F}$ & 1 \\
\hline & pigs & $\mathrm{E}$ & 3 \\
\hline
\end{tabular}

\subsection{Antimicrobial Resistance among Generic E. coli, Broad-and Spectrum $\beta$-Lastamase E. coli, and STEC}

Antibiotic-resistant generic E. coli isolates were observed most often in pig (83\%) and cattle (56\%) feces, as well as feather swabs (66\%; Table 5). The majority of AMR E. coli isolates from animal feces and hide/feather swabs $(n=69)$ were resistant to only one class of antibiotics. Six isolates were resistant to 3 or 4 classes. Isolates resistant $\geq 5$ antibiotic classes were only obtained from cattle feces. Half of the AMR generic E. coli isolated from wooden hand rails were MDR. Conversely, AMR E. coli collected from metal hand rails were only resistant to tetracycline.

All broad- and extended-spectrum $\beta$-lastamase E. coli were isolated from animal feces, except for three MDR isolates from wooden hand rails (Table 5). More than $80 \%$ of the $\beta$-lastamase-producing $E$. coli were MDR, originating from pig (35\%), sheep and goat (31\%), and cattle (18\%) feces.

Of the 66 STEC isolates collected in this study, 38\% were resistant to antimicrobials (Table 5). All AMR STEC obtained from wooden hand rails were MDR, while those from feces and hides/feathers were resistant to only one class of antibiotic. One isolate from chicken feathers was the exception as it was resistant to five antibiotic classes.

\subsection{Phylogroups among Generic E. coli and $\beta$-Lactamase-Producing E. coli}

All E. coli phylogroups were represented among the generic and $\beta$-lactamase-producing E. coli isolates with the majority belonging to phylogroup B1 (Table 6). Phylogroup A was also consistently observed (11-13\% of isolates) among all sample types. In addition to phylogroup B1, phylogroup E was also common in ESBL-producing E. coli (37\%, Table 6). Isolates belonging to phylogroup C were observed among $0-6 \%$ of isolates from each sample type, while the remaining phylogroups (i.e., B2, D, $\mathrm{F}$, and clades I/II) were uncommon $(\leq 3 \%)$ among both generic and ESBL-producing E. coli. 
Table 5. Frequency of antimicrobial resistance (AMR) patterns among generic E. coli $(n=425)$, broad- and extended-spectrum $\beta$-lactamase $E$. coli $(n=59)$, and STEC $(n$ =66) isolates from animal feces, hide/feather swabs, and hand rails.

\begin{tabular}{|c|c|c|c|c|c|c|c|c|c|c|c|c|c|c|}
\hline \multirow[b]{2}{*}{ 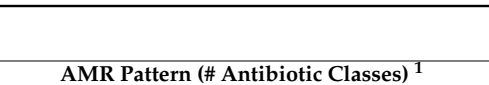 } & \multicolumn{6}{|c|}{ Composite Fecal Samples } & \multicolumn{7}{|c|}{ Hide or Feather Swabs } & \multirow{2}{*}{ 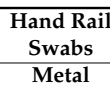 } \\
\hline & Cattle & Sheep/Goats & Pigs & Horses/Donkeys & Llamas & Birds & Cattle & Sheep/Goats & Pigs & Horses/Donkeys & Llamas & Birds & Wood & \\
\hline Generic E. coli $(n=\#$ isolates $)$ & $n=25$ & $n=108$ & $n=35$ & $n=52$ & $n=18$ & $n=18$ & $n=12$ & $n=33$ & $n=8$ & $n=14$ & $n=9$ & $n=7$ & $n=66$ & $n=20$ \\
\hline $\begin{array}{l}\text { AMC-AMP-CAZ-CFX-CHL-CRO-CTX-GEN-KAN } \\
\text {-STREP-TET-TMS (6) }\end{array}$ & $1^{2}$ & - & - & - & - & - & - & - & - & - & - & - & - & - \\
\hline $\begin{array}{l}\text { AMC-AMP-CAZ-CFX-CHL-CRO-CTX-KAN } \\
\text {-STREP-TET-TMS (6) }\end{array}$ & 2 & - & - & - & - & - & - & - & - & - & - & - & - & - \\
\hline $\begin{array}{l}\text { AMC-AMP-CAZ-CFX-CHL-CRO-CTX-GEN-KAN } \\
\text {-STREP-TET (5) }\end{array}$ & 2 & - & - & - & - & - & - & - & - & - & - & - & - & - \\
\hline AMC-AMP-KAN-STREP-TET (4) & - & 3 & - & - & - & - & - & - & - & - & - & - & - & - \\
\hline AMP-STREP-TET-TMS (4) & - & - & - & - & 1 & - & - & - & - & - & - & - & 4 & - \\
\hline CHL-STREP-TET-TMS (4) & - & - & - & - & - & - & - & - & - & - & - & - & 1 & - \\
\hline CHL-STREP-TET (3) & - & - & $1^{3}$ & - & - & - & - & - & - & - & - & - & 2 & - \\
\hline CHL-STREP-TMS (3) & - & - & - & $1^{4}$ & - & - & - & - & - & - & - & - & - & - \\
\hline CTX-CRO (1) & - & - & - & - & - & - & - & - & - & - & 1 & - & - & - \\
\hline KAN-STREP (1) & - & 1 & - & 3 & - & - & - & - & - & - & - & - & - & - \\
\hline STREP-TET (2) & 1 & 3 & 6 & - & - & - & - & 1 & - & 2 & - & 2 & - & - \\
\hline STREP-TMS (2) & - & - & - & 1 & - & - & - & - & - & - & - & - & - & - \\
\hline TET-TMS (2) & - & - & 1 & - & - & - & - & - & - & - & - & - & - & - \\
\hline STREP (1) & - & 1 & 5 & - & - & - & - & - & - & - & - & - & - & - \\
\hline TET (1) & 2 & 10 & 2 & 4 & - & 5 & - & 9 & 1 & 1 & - & - & 7 & 3 \\
\hline AMP (1) & 1 & - & 1 & - & 4 & - & - & 1 & - & - & - & - & - & - \\
\hline Susceptible isolates & 16 & 90 & 19 & 43 & 13 & 13 & 0 & 22 & 7 & 11 & 8 & 5 & 52 & 17 \\
\hline General resistance prevalence per sample $(\%)^{5}$ & $5 / 9(56)$ & 9/36 (25) & $10 / 12(83)$ & $4 / 18(22)$ & $3 / 6(50)$ & $3 / 7(43)$ & $0 / 4(0)$ & $5 / 15(33)$ & $1 / 5(20)$ & $2 / 5(40)$ & $1 / 3(33)$ & $2 / 3(66)$ & $8 / 28(29)$ & $1 / 18(6)$ \\
\hline $\begin{array}{l}\text { AMC-AMP-CAZ-CFX-CHL-CIP-CRO-CTX-NAL } \\
\text {-STREP-TET-TMS (7) }\end{array}$ & - & 1 & - & - & - & - & - & - & - & - & - & - & - & - \\
\hline $\begin{array}{l}\text { AMC-AMP-CAZ-CFX-CHL-CRO-CTX-GEN } \\
\text {-KAN-STREP-TET (5) }\end{array}$ & 6 & - & - & - & - & - & - & - & - & - & - & - & - & - \\
\hline $\begin{array}{l}\text { AMP-CAZ-CFX-CHL-CIP-CRO-CTX-NAL } \\
\text {-STREP-TET-TMS (6) }\end{array}$ & - & 1 & - & - & - & - & - & - & - & - & - & - & - & - \\
\hline $\begin{array}{l}\text { AMC-AMP-CAZ-CFX-CHL-CRO-CTX-NAL } \\
\text {-STREP-TET (6) }\end{array}$ & - & 6 & - & - & - & - & - & - & - & - & - & - & 3 & - \\
\hline $\begin{array}{l}\text { AMC-AMP-CAZ-CFX-CHL-CRO-CTX } \\
\text {-STREP-TET-TMS (6) }\end{array}$ & - & 3 & 4 & - & - & - & - & - & - & - & - & - & - & - \\
\hline $\begin{array}{l}\text { AMP-CAZ-CHL-CIP-CRO-CTX-NAL } \\
\text {-STREP-TET-TMS (6) }\end{array}$ & - & - & $1^{6}$ & - & - & - & - & - & - & - & - & - & - & - \\
\hline $\begin{array}{l}\text { AMC-AMP-CAZ-CFX-CHL-CRO-CTX } \\
\text {-STREP-TET (5) }\end{array}$ & 3 & 3 & 2 & 3 & - & - & - & - & - & - & - & - & - & - \\
\hline $\begin{array}{l}\text { AMP-CHL-CIP-CRO-CTX-NAL-STREP-TET } \\
\text {-TMS (6) }\end{array}$ & - & 1 & 2 & - & - & 2 & - & - & - & - & - & - & - & - \\
\hline AMC-AMP-CAZ-CFX-CRO-CTX-STREP-TET (4) & - & - & 2 & - & - & - & - & - & - & - & - & - & - & - \\
\hline AMP-CAZ-CHL-CRO-CTX-STREP-TET-TMS (5) & - & - & 1 & - & - & - & - & - & - & - & - & - & - & - \\
\hline AMC-AMP-CAZ-CFX-CRO-CTX-TET (3) & - & - & 4 & - & - & - & - & - & - & - & - & - & - & - \\
\hline AMC-AMP-CFX-CHL-CTX-STREP-TET (5) & - & - & 1 & - & - & - & - & - & - & - & - & - & - & - \\
\hline AMC-AMP-CAZ-CFX-CRO-CTX (2) & - & 6 & - & - & - & - & - & - & - & - & - & - & - & - \\
\hline AMP-CRO-CTX-TET (2) & - & - & - & - & - & 1 & - & - & - & - & - & - & - & - \\
\hline AMP-CRO-CTX (1) & 3 & - & - & - & - & - & - & - & - & - & - & - & - & - \\
\hline
\end{tabular}


Table 5. Cont

\begin{tabular}{|c|c|c|c|c|c|c|c|c|c|c|c|c|c|c|}
\hline & \multicolumn{6}{|c|}{ Composite Fecal Samples } & \multicolumn{7}{|c|}{ Hide or Feather Swabs } & \multirow{2}{*}{$\begin{array}{c}\text { Hand Rail } \\
\text { Swabs } \\
\text { Metal }\end{array}$} \\
\hline 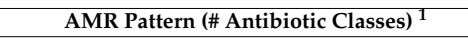 & Cattle & Sheep/Goats & Pigs & Horses/Donkeys & Llamas & Birds & Cattle & Sheep/Goats & Pigs & Horses/Donkeys & Llamas & Birds & Wood & \\
\hline Generic $E$. coli $(n=\#$ isolates $)$ & $n=25$ & $n=108$ & $n=35$ & $n=52$ & $n=18$ & $n=18$ & $n=12$ & $n=33$ & $n=8$ & $n=14$ & $n=9$ & $n=7$ & $n=66$ & $n=20$ \\
\hline General resistance prevalence per sample $(\%)^{5}$ & $4 / 9(44)$ & $7 / 36(19)$ & $7 / 12(58)$ & $1 / 18(6)$ & $0 / 0(0)$ & $1 / 7(14)$ & $0 / 0(0)$ & $0 / 0(0)$ & $0 / 0(0)$ & $0 / 0(0)$ & $0 / 0(0)$ & $0 / 0(0)$ & $1 / 28(4)$ & $0 / 0(0)$ \\
\hline $\begin{array}{l}\text { AMC-AMP-CAZ-CFX-CHL-CRO-CTX-GEN } \\
\text {-STREP-TET (5) }\end{array}$ & - & - & - & - & - & - & - & - & - & - & - & 1 & - & - \\
\hline CHL-STREP-TET-TMS (4) & - & - & - & - & - & - & - & - & - & - & - & - & 1 & - \\
\hline STREP-TET-TMS (3) & - & - & - & - & - & - & - & - & - & - & - & - & 3 & - \\
\hline KAN-STREP (1) & 2 & 1 & - & 6 & - & - & - & - & - & - & - & - & - & - \\
\hline TET (1) & - & 1 & 4 & 4 & - & - & - & 1 & - & - & - & 1 & - & - \\
\hline Susceptible isolates & 6 & 10 & 3 & 4 & 7 & 3 & 0 & 0 & 2 & 0 & 0 & 1 & 5 & 0 \\
\hline General resistance prevalence per sample $(\%)^{5}$ & 1/9 (11) & $2 / 36(6)$ & $3 / 12(25)$ & $5 / 18(28)$ & $0 / 6(0)$ & $0 / 7(0)$ & $0 / 4(0)$ & 1/15 (7) & $0 / 5(0)$ & $0 / 5(0)$ & $0 / 3(0)$ & $2 / 3(66)$ & $2 / 28(7)$ & $0 / 18(0)$ \\
\hline
\end{tabular}

${ }^{1} \mathrm{AMC}$, amoxicillin/clavulonic acid; AMP, ampicillin; CAZ, ceftazidime; CHL, chloramphenicol; CIP, ciprofloxacin; CFX, cefoxitin; CRO, ceftriaxone; CTX, cefotaxime; GEN, gentamycin;

KAN, kanamycin; NAL, naladixic acid; STREP, streptomycin; TET, tetracycline; TMS, trimethoprim/sulfamethoxazole. ${ }^{2}$ Intermediate resistance to CAZ and GEN. ${ }^{3}$ Intermediate resistance to STREP. ${ }^{4}$ Intermediate resistance to CHL and TMS. ${ }^{5}$ \# samples with $\geq 1$ AMR isolate / total \# samples. ${ }^{6}$ Intermediate resistance to CAZ. 
Table 6. Assigned phylogenetic groups (A, B1, B2, C, D, E, F, Clades I or II, or Unknown) of E. coli strains from different hosts based on multiplex PCR of 4 genetic markers ( $\operatorname{arp} A, \operatorname{chuA}$, yjaA, TspE4.C2). Numbers indicate the number of bacterial isolates obtained per species. An overall representation of bacterial isolates per phylogroup is given as a percentage out of the total number of isolates collected.

\begin{tabular}{|c|c|c|c|c|c|c|c|c|c|c|}
\hline & Phylogroup & Cattle & Sheep/Goats & Pigs & Horses/Donkeys & Llamas & Birds & Wood & Metal & $\%$ Isolates \\
\hline \multirow{9}{*}{$\begin{array}{l}\text { Generic } E \text {. coli isolates from } \\
\quad \text { animal feces }(n=256)\end{array}$} & $\mathrm{A}$ & 5 & 15 & 5 & 1 & 1 & 2 & NA & NA & 11 \\
\hline & B1 & 17 & 67 & 25 & 33 & 13 & 13 & NA & NA & 66 \\
\hline & B2 & 0 & 0 & 0 & 0 & 0 & 0 & NA & NA & 0 \\
\hline & $\mathrm{C}$ & 0 & 7 & 0 & 7 & 0 & 1 & NA & NA & 6 \\
\hline & $\mathrm{D}$ & 0 & 1 & 1 & 3 & 0 & 0 & NA & NA & 2 \\
\hline & E & 2 & 7 & 2 & 3 & 4 & 1 & NA & NA & 7 \\
\hline & $\mathrm{F}$ & 0 & 0 & 0 & 0 & 0 & 0 & NA & NA & 0 \\
\hline & Clade I or II & 0 & 3 & 1 & 0 & 0 & 0 & NA & NA & 2 \\
\hline & Unknown & 1 & 8 & 1 & 5 & 0 & 1 & $\mathrm{NA}$ & NA & 6 \\
\hline \multirow{9}{*}{$\begin{array}{l}\text { Generic } E \text {. coli isolates from } \\
\text { animal hide swabs }(n=83)\end{array}$} & $\mathrm{A}$ & 1 & 1 & 1 & 0 & 0 & 7 & NA & NA & 12 \\
\hline & B1 & 8 & 23 & 6 & 12 & 5 & 0 & NA & NA & 65 \\
\hline & B2 & 0 & 0 & 0 & 0 & 2 & 0 & $\mathrm{NA}$ & NA & 2 \\
\hline & $\mathrm{C}$ & 1 & 3 & 1 & 0 & 0 & 0 & NA & NA & 6 \\
\hline & $\mathrm{D}$ & 0 & 0 & 0 & 1 & 0 & 0 & NA & NA & 1 \\
\hline & E & 1 & 3 & 0 & 1 & 2 & 0 & NA & NA & 8 \\
\hline & $\mathrm{F}$ & 0 & 0 & 0 & 0 & 0 & 0 & NA & NA & 0 \\
\hline & Clade I or II & 0 & 1 & 0 & 0 & 0 & 0 & NA & NA & 1 \\
\hline & Unknown & 1 & 2 & 0 & 0 & 0 & 0 & NA & NA & 4 \\
\hline \multirow{9}{*}{$\begin{array}{l}\text { Generic } E \text {. coli isolates from } \\
\text { hand rail swabs }(n=86)\end{array}$} & $\mathrm{A}$ & NA & NA & NA & NA & NA & NA & 8 & 3 & 13 \\
\hline & B1 & NA & NA & NA & NA & NA & NA & 39 & 12 & 59 \\
\hline & B2 & NA & NA & NA & NA & NA & NA & 0 & 0 & 0 \\
\hline & $\mathrm{C}$ & NA & NA & NA & NA & NA & NA & 2 & 0 & 2 \\
\hline & $\mathrm{D}$ & NA & NA & NA & NA & NA & NA & 0 & 0 & 0 \\
\hline & $\mathrm{E}$ & NA & NA & NA & NA & NA & NA & 14 & 5 & 22 \\
\hline & F & NA & NA & NA & NA & NA & NA & 0 & 0 & 0 \\
\hline & Clade I or II & NA & NA & NA & NA & NA & NA & 3 & 0 & 3 \\
\hline & Unknown & NA & NA & NA & NA & NA & NA & 0 & 0 & 0 \\
\hline \multirow{9}{*}{$\begin{array}{l}\text { B-lactamase -producing } E \text {. } \\
\text { coli isolates from feces and } \\
\text { hand rail swabs }(n=59)\end{array}$} & $\mathrm{A}$ & 0 & 0 & 3 & 0 & 0 & 1 & 0 & 0 & 7 \\
\hline & B1 & 4 & 12 & 7 & 0 & 0 & 1 & 0 & 0 & 41 \\
\hline & B2 & 0 & 0 & 0 & 0 & 0 & 0 & 0 & 0 & 0 \\
\hline & $\mathrm{C}$ & 0 & 0 & 0 & 0 & 0 & 0 & 0 & 0 & 0 \\
\hline & $\mathrm{D}$ & 0 & 0 & 1 & 0 & 0 & 0 & 0 & 0 & 1.5 \\
\hline & $\mathrm{E}$ & 4 & 9 & 3 & 3 & 0 & 0 & 3 & 0 & 37 \\
\hline & F & 0 & 0 & 0 & 1 & 0 & 0 & 0 & 0 & 1.5 \\
\hline & Clade I or II & 0 & 0 & 0 & 0 & 0 & 0 & 0 & 0 & 0 \\
\hline & Unknown & 4 & 0 & 2 & 0 & 0 & 1 & 0 & 0 & 12 \\
\hline
\end{tabular}




\section{Discussion}

\subsection{Survey}

To our knowledge, this is the first survey of hygienic practices employed in Canadian livestock fairs and petting zoos. Several investigations from the U.S. have identified contact with animals and their feces, poor hand-washing compliance, and lack of or inadequately equipped hand-washing facilities as factors that precipitate disease outbreaks [31,32]. Restrictions on food and drink in animal areas, frequent and immediate removal of animal feces, provision of hand-washing and hand-sanitizing facilities, and more frequent surface disinfection are practices that could also reduce health risks in Canadian petting zoos and fairs. The U.S. National Association of State Public Health Veterinarians released a report of recommendations to prevent disease associated with animals in public settings [33]. A similar document outlining standard operating, cleaning, and sanitation procedures from a Canadian authority would provide a beneficial resource for agricultural fair coordinators and operators of public animal venues in Canada. However, without official policies and enforcement in place, compliance with recommendations remains voluntary.

\subsection{Prevalence of $\mathrm{O} 157$ and Non-O157 STEC}

Due to the low infective dose of STEC O157:H7 and the severity of illness that can develop, particularly in young children, it is the pathogen of utmost concern for petting zoos [2,5]. Previous studies reported a high prevalence of STEC O157 at county fairs in the U.S. (75-97\%) with cattle being the primary host (11\% host prevalence), followed by sheep and goats (3.6\%), and swine $(1.2 \%)$ [34]. In the current study, STEC O157 was isolated once from goat feces. Cattle were not common at the petting zoos sampled in this study; hence, only nine composite cattle fecal samples were collected compared to 36 from sheep and goats.

Pathogenic E. coli $\mathrm{O} 157$ can survive in the environment outside the animal host for weeks to months [4], and viable E. coli O157 have been cultured from fairgrounds and petting zoos months after outbreaks have occurred $[35,36]$. Hand rails can become contaminated at fairs and petting zoos and have been implicated as sources of E. coli O157 following outbreaks [36,37]. Although E. coli O157 was not isolated from hand rail swabs in the current study, non-O157 STEC were, even from hand rails that were reportedly sanitized daily.

To our knowledge, this is the first study to examine the prevalence of the top 6 non-O157 STEC serogroups in animals at fairs and petting zoos. Most studies of non-O157 STEC report prevalence in food animals, of which cattle, small ruminants, pigs, and poultry are important sources $[5,38]$. However, non-O157 STEC are widespread and have been isolated from numerous non-food domestic and wild animals [5,32]. According to previous studies, llamas, horses, and donkeys are not considered important STEC reservoirs [4,39]. In the current study, STEC were isolated from llama feces from the same petting zoo on two separate occasions, and from horse and donkey feces collected from two different petting zoos (Table 2). The relatively high prevalence of STEC in fecal samples from these animals may reflect animal-to-animal transmission, considering the proximity of housing within petting zoos and fairs [40]. This possibility is supported by the isolation of STEC from multiple animal species at the same petting zoos that possessed the same serotype, virulence, and AMR phenotypes.

Research of STEC loads on hides is primarily focused on cattle since hides represent a major source of carcass contamination during meat processing [5]. No research comparable to the current study for STEC prevalence on the hides, fur, or feathers of other animal species could be identified. Considering that contact with animals at petting zoos is primarily from active contact with hides and feather, findings of the current study emphasize the importance of supplying hand-washing facilities within petting zoo areas. 


\subsection{Prevalence of Salmonella, MRSA, Campylobacter, Cryptosporidium and Giardia}

Research suggests that the prevalence of Salmonella is lower in Canada and the northern U.S. than the southern U.S. [41,42] and that management practices may have a profound effect on the transmission and persistence of salmonellae within an integrated production system [43]. Animals sampled within this study were not a part of the production system, coupled with the small sample size, the lack of Salmonella in this study was perhaps not surprising.

Pigs are a reservoir for $S$. aureus, and MRSA have been isolated from pigs at an agricultural fair in the U.S. [44]. Though pigs at fairs in Canada have not previously been tested, other studies indicate MRSA are common in pigs on Canadian production farms [45]. Since pigs were not frequently encountered at the petting zoos visited in this study resulting in a small sample size, it is perhaps not surprising that none of the nasal swabs were positive for MRSA.

Compared to the other pathogens in this study, Campylobacter is thought to cause the highest number of human illnesses due to transmission from animals [3]. Healthy poultry, cattle, swine, and sheep are considered important reservoirs for this organism [20,46]. We isolated Campylobacter spp. from sheep and goats, pigs, horses and donkeys, and birds. Isolation of Campylobacter from healthy horses has been reported, but much less frequently than for other host species [47]. A similar study isolated Campylobacter spp. from cattle, sheep, goats, and chickens at a petting zoo in California [11]. In contrast to the high proportion of Campylobacter-positive samples found in our study, a meta-analysis estimate of Campylobacter prevalence among petting zoo animals was determined to be 6.5\% [48]. The meta-analysis was derived from only seven studies, as there is a lack of research on Campylobacter at fairs and petting zoos in Canada. Furthermore, non-jejuni/coli species are of uncertain significance to human health [46]. Additional work is required to fully assess the risk of Campylobacter infection from petting zoos and fairs in Canada.

Cryptosporidium was only detected in sheep and goat feces, with the Cryptosporidium bovis identified in goat/sheep fecal samples occasionally being associated with human illness [49]. A zoonotic assemblage of Giardia intestinalis (assemblage A) was detected in cattle, horses, and donkeys. Neither pathogen was detected in feces of petting zoo animals in a similar U.S. study [11].

\subsection{AMR, Broad-and Extended-Spectrum $\beta$-Lactamase- E. coli}

AMR E. coli were observed among all animal groups and sample types tested. Collectively, 22\% of the generic E. coli isolates from this study were resistant to one or more antimicrobials. Other studies report varied rates of resistance in generic E. coli from different animal groups in Canada [50,51].

Very few isolates $(1.3 \%)$ were resistant to the critically important 3rd generation cephalosporins or quinolones. No isolates were resistant to imipenem. Resistance to tetracycline was most common and has been widely reported in dairy and beef cattle, small ruminants, pigs, poultry, equines, and petting zoo animals [11,51-54]. Resistance to ampicillin, streptomycin and kanamycin is also common in E. coli from animal feces [11,52,54].

Although MDR among generic E. coli and STEC was not common, MDR E. coli including STEC were isolated from wooden hand rails, possibly posing an even greater risk of transmission within the petting zoo than bacteria directly associated with animal feces. Although these isolates may not be pathogenic, they may be carriers of AMR genes that can be readily exchanged with other bacteria [50].

Since the late 1990s, ESBL-producing E. coli have emerged globally [10]. Early $\beta$-lactamase-producing E. coli possessed mainly TEM and SHV $\beta$-lactamases, while the significance of CTX-M-type enzymes has since increased and currently represents the most common type in humans [10]. Infection with $\beta$-lactamase-producing E. coli is of concern because of limited therapeutic options [10]. Only the most common broad-spectrum $\beta$-lactamase and ESBL genotypes were screened for in this study, suggesting the isolates with unidentified genotypes (>60\%) may have been of less common $\beta$-lactamase types. The detection of broad- and extended-spectrum $\beta$-lactamase-producing $E$. coli in this study is to be considered a preliminary screening. Future studies must also include phenotypic confirmation of putative ESBL-producing E. coli isolates. 


\subsection{Phylogroups}

The clonal population structure of E. coli has enabled assignation of isolates to phylogroups (A, B1, B2, C, D, E, F, and cryptic clades) $[29,30]$ which vary in host association, presence of virulence factors, and persistence in the non-host environment [29]. Distinct ecological niches characterize the different phylogroups. For example, phylogroups A and B2 are predominant in humans, whereas phylogroups A and B1 are more common in animals [55].

Although representatives from every phylogroup were identified, the most common phylogroup was B1. These results are consistent with previous studies which have identified a higher proportion of B1 among cows, goats, and sheep [56]. Our results suggest that E. coli from phylogroups A, B1, and $\mathrm{E}$ are the most common in petting zoo animals and their environments. From a human health perspective, E. coli responsible for mild and chronic diarrhea are found throughout the phylogenetic tree, whereas E. coli that cause more severe pathologies are frequently restricted to phylogroups A, B1, and E [55]. Whether any of the isolates collected in the current study could cause mild or severe illness in humans remains unknown, but measures that reduce the risk of ingestion of these bacteria [2], as well as cleaning and sanitizing procedures to decrease environmental persistence undoubtedly have merit.

\section{Conclusions}

Effective hygienic preventative measures within fairs and petting zoos rely on assessing risks for human exposure to zoonotic pathogens. Despite the limited number of petting zoos and samples in this study, we have shown that some zoonotic pathogens are clearly present within petting zoo animals and their environments. Of concern are STEC and Campylobacter species based on their prevalence and/or potential to cause serious illness. In addition, a variety of virulence genes and AMR E. coli were present in a variety of animal species and on hand rails suggesting the potential for transfer of these elements to other E. coli and/or pathogens.

Certain inadequacies are evident within the operation of animal areas accessible to the public at fairs in Canada, such as the absence of hand-washing facilities at many venues. Sanitization of surfaces within petting zoos and livestock pavilions is largely sporadic, with some venues lacking sanitization procedures. These data should not deter the public from visiting agricultural fairs but should serve as reminder to event operators and visitors alike, of the importance of good hygiene and sanitation practices. Materials used to construct animal areas and pens should be non-porous, such as metal rather than wood, allowing more effective sanitization. Fair and petting zoo operators are encouraged to consult the recommendations outlined by the National Association of State Public Health Veterinarians. Ongoing efforts to reduce risks of human exposure to zoonotic pathogens can help to ensure children and adults continue to enjoy agricultural events and the educational opportunities they provide.

Further research is necessary to better understand the human health risk associated with fairs and petting zoos. Future studies should include identification of plasmids in E. coli strains to identify the dissemination potential and possible transfer of AMR genes to other E. coli strains and other members of Enterobacteriaceae. Multilocus Sequence Typing (MLST) of E. coli strains could be used to determine clonality and animal/human dissemination potential. Additionally, the inclusion of animal and human clinical isolates could be used to identify common characteristics between clinical isolates and those collected from petting zoos.

Author Contributions: Conceptualization, C.C., K.S., T.A.M. and C.N.B.; Methodology, C.C., T.A.M., N.N., L.T. and C.J.; Formal Analysis, C.C. and K.S.; Investigation, C.C. and C.J.; Resources, K.S., T.A.M. and N.N.; Data Curation, C.C. and K.S.; Writing-Original Draft Preparation, C.C.; Writing-Review and Editing, K.M., L.T., C.J., C.N.B., K.S. and T.A.M.; Visualization, C.C., C.J. and K.M.; Supervision, T.A.M.; Project Administration, C.C. and T.A.M.; Funding Acquisition, K.O.

Funding: This study was made possible by the Canadian Association of Fairs, Agriculture and Agri-Food Canada Growing Forward 2 Program, and Alberta Agriculture and Forestry. 
Acknowledgments: The authors would like to express their appreciation to: Karen Oliver, Executive Director of Canadian Association of Fairs; Susanne Trapp, Yidong Graham, Sarah-Jo Paquette, and Jessica Franken for their technical assistance; Graham Banting for his assistance and expertise with Cryptosporidium and Giardia detection; and Michael Harker, Senior Partner with Enigma Research Corporation, for his expertise in conducting the survey.

Conflicts of Interest: The authors declare no conflict of interest.

\section{References}

1. Weese, J.S.; McCarthy, L.; Mossop, M.; Martin, H.; Lefebvre, S. Observation of practices at petting zoos and the potential impact on zoonotic disease transmission. Clin. Infect. Dis. 2007, 45, 10-15. [CrossRef] [PubMed]

2. Conrad, C.C.; Stanford, K.; Narvaez-Bravo, C.; Callaway, T.; McAllister, T. Farm fairs and petting zoos: A review of animal contact as a source of zoonotic enteric disease. Foodborne Pathog. Dis. 2017, 14, 59-73. [CrossRef] [PubMed]

3. Hale, C.R.; Scallan, E.; Cronquist, A.B.; Dunn, J.; Smith, K.; Robinson, T.; Lathrop, S.; Tobin-D'Angelo, M.; Clogher, P. Estimates of enteric illness attributable to contact with animals and their environments in the United States. Clin. Infect. Dis. 2012, 54, 472-479. [CrossRef] [PubMed]

4. Ferens, W.A.; Hovde, C.J. Escherichia coli O157:H7: Animal reservoir and sources of human infection. Foodborne Pathog. Dis. 2011, 8, 465-487. [CrossRef] [PubMed]

5. Baker, C.A.; Rubinelli, P.M.; Park, S.H.; Carbonero, F.; Ricke, S.C. Shiga toxin-producing Escherichia coli in food: Incidence, ecology, and detection strategies. Food Control 2016, 59, 407-419. [CrossRef]

6. Bolton, D.J. Campylobacter virulence and survival factors. Food Microbiol. 2015, 48, 99-108. [CrossRef] [PubMed]

7. Erdozain, G.; KuKanich, K.; Chapman, B.; Powell, D. Observation of public health risk behaviours, risk communication and hand hygiene at Kansas and Missouri petting zoos. Zoonoses public health 2013, 60, 304-310. [CrossRef] [PubMed]

8. Anderson, M.E.; Weese, J.S. Video observation of hand hygiene practices at a petting zoo and the impact of hand hygiene interventions. Epidemiol. Infect. 2012, 140, 182-190. [CrossRef] [PubMed]

9. Collignon, P.; Powers, J.H.; Chiller, T.M.; Aidara-Kane, A.; Aarestrup, F.M. World health organization ranking of antimicrobials according to their importance in human medicine: A critical step for developing risk management strategies for the use of antimicrobials in food production animals. Clin. Infect. Dis. 2009, 49, 132-141. [CrossRef] [PubMed]

10. Ewers, C.; Bethe, A.; Semmler, T.; Guenther, S.; Wieler, L.H. Extended-spectrum beta-lactamase-producing and ampc-producing Escherichia coli from livestock and companion animals, and their putative impact on public health: A global perspective. Clin. Microbiol. Infect. 2012, 18, 646-655. [CrossRef] [PubMed]

11. Roug, A.; Byrne, B.A.; Conrad, P.A.; Miller, W.A. Zoonotic fecal pathogens and antimicrobial resistance in county fair animals. Comp. Immunol. Microbiol. Infect. Dis. 2013, 36, 303-308. [CrossRef] [PubMed]

12. Brooks, J.T.; Sowers, E.G.; Wells, J.G.; Greene, K.D.; Griffin, P.M.; Hoekstra, R.M.; Strockbine, N.A. Non-O157 shiga toxin-producing Escherichia coli infections in the United States, 1983-2002. J. Infect. Dis. 2005, 192, 1422-1429. [CrossRef] [PubMed]

13. Stanford, K.; Johnson, R.P.; Alexander, T.W.; McAllister, T.A.; Reuter, T. Influence of season and feedlot location on prevalence and virulence factors of seven serogroups of Escherichia coli in feces of western-Canadian slaughter cattle. PloS ONE 2016, 11, e0159866. [CrossRef] [PubMed]

14. Conrad, C.C.; Stanford, K.; McAllister, T.A.; Thomas, J.; Reuter, T. Further development of sample preparation and detection methods for $\mathrm{O} 157$ and the top 6 non-O157 STEC serogroups in cattle feces. J. Microbiol. Methods 2014, 105, 22-30. [CrossRef] [PubMed]

15. Machado, J.; Grimont, F.; Grimont, P.A. Identification of Escherichia coli flagellar types by restriction of the amplified flic gene. Res. Microbiol. 2000, 151, 535-546. [CrossRef]

16. Bej, A.K.; DiCesare, J.L.; Haff, L.; Atlas, R.M. Detection of Escherichia coli and Shigella spp. In water by using the polymerase chain reaction and gene probes for uid. Appl. Environ. Microbiol. 1991, 57, $1013-1017$. [PubMed] 
17. Maheux, A.F.; Picard, F.J.; Boissinot, M.; Bissonnette, L.; Paradis, S.; Bergeron, M.G. Analytical comparison of nine PCR primer sets designed to detect the presence of Escherichia coli/Shigella in water samples. Water Res. 2009, 43, 3019-3028. [CrossRef] [PubMed]

18. Dallenne, C.; Da Costa, A.; Decre, D.; Favier, C.; Arlet, G. Development of a set of multiplex PCR assays for the detection of genes encoding important beta-lactamases in enterobacteriaceae. J. Antimicrob. Chemother. 2010, 65, 490-495. [CrossRef] [PubMed]

19. Jokinen, C.C.; Koot, J.M.; Carrillo, C.D.; Gannon, V.P.; Jardine, C.M.; Mutschall, S.K.; Topp, E.; Taboada, E.N. An enhanced technique combining pre-enrichment and passive filtration increases the isolation efficiency of Campylobacter jejuni and Campylobacter coli from water and animal fecal samples. J. Microbiol. Methods 2012, 91, 506-513. [CrossRef] [PubMed]

20. LaGier, M.J.; Joseph, L.A.; Passaretti, T.V.; Musser, K.A.; Cirino, N.M. A real-time multiplexed PCR assay for rapid detection and differentiation of Campylobacter jejuni and Campylobacter coli. Mol. Cell Probes 2004, 18, 275-282. [CrossRef] [PubMed]

21. Iwamoto, T.; Tani, K.; Nakamura, K.; Suzuki, Y.; Kitagawa, M.; Eguchi, M.; Nasu, M. Monitoring impact of in situ biostimulation treatment on groundwater bacterial community by DGGE. FEMS Microbiol. Ecol. 2000, 32, 129-141. [CrossRef] [PubMed]

22. Kawai, M.; Matsutera, E.; Kanda, H.; Yamaguchi, N.; Tani, K.; Nasu, M. 16s ribosomal DNA-based analysis of bacterial diversity in purified water used in pharmaceutical manufacturing processes by PCR and denaturing gradient gel electrophoresis. Appl. Environ. Microbiol. 2002, 68, 699-704. [CrossRef] [PubMed]

23. Ruecker, N.J.; Hoffman, R.M.; Chalmers, R.M.; Neumann, N.F. Detection and resolution of Cryptosporidium species and species mixtures by genus-specific nested PCR-restriction fragment length polymorphism analysis, direct sequencing, and cloning. Appl. Environ. Microbiol. 2011, 77, 3998-4007. [CrossRef] [PubMed]

24. Ruecker, N.J.; Matsune, J.C.; Lapen, D.R.; Topp, E.; Edge, T.A.; Neumann, N.F. The detection of Cryptosporidium and the resolution of mixtures of species and genotypes from water. Infect. Genet. Evol. 2013, 15, 3-9. [CrossRef] [PubMed]

25. Hopkins, R.M.; Meloni, B.P.; Groth, D.M.; Wetherall, J.D.; Reynoldson, J.A.; Thompson, R.C.A. Ribosomal RNA sequencing reveals differences between the genotypes of Giardia isolates recovered from humans and dogs living in the same locality. J. Parasitol. 1997, 83, 44-51. [CrossRef] [PubMed]

26. Smith, A.; Neumann, N.; Banting, G.; Klein, C.; Liccioli, S.; Massolo, A. Giardia spp. And cryptosporidium spp. Infecting urban dogs and coyote pose a low risk for public. Zoonoses 2017. Under Review.

27. Clinical and Laboratory Standards Institute (CLSI). Performance Standards for Antimicrobial Disc Susceptibility Tests; Approved Standard-Twelfth Edition M02-A12; CLSI: Wayne, PA, USA, 2015.

28. Clinical and Laboratory Standards Institute (CLSI). Performance Standards for Antimicrobial Susceptibility Testing; M100-S26; CLSI: Wayne, PA, USA, 2016.

29. Franz, E.; Veenman, C.; van Hoek, A.; Husman, A.d.R.; Blaak, H. Pathogenic Escherichia coli producing extended-spectrum $\beta$-lactamases isolated from surface water and wastewater. Sci. Rep. 2015, 5, 14372. [CrossRef] [PubMed]

30. Clermont, O.; Christenson, J.K.; Denamur, E.; Gordon, D.M. The Clermont Escherichia coli phylo-typing method revisited: Improvement of specificity and detection of new phylo-groups. Environ. Microbiol. Rep. 2013, 5, 58-65. [CrossRef] [PubMed]

31. Bender, J.B.; Shulman, S.A. Reports of zoonotic disease outbreaks associated with animal exhibits and availability of recommendations for preventing zoonotic disease transmission from animals to people in such settings. J. Am. Vet. Med. Assoc. 2004, 224, 1105-1109. [CrossRef] [PubMed]

32. LeJeune, J.T.; Davis, M.A. Outbreaks of zoonotic enteric disease associated with animal exhibits. J. Am. Vet. Med. Assoc. 2004, 224, 1440-1445. [CrossRef] [PubMed]

33. National Association of State Public Health Veterinarians. Compendium of measures to prevent disease associated with animals in public settings. J. Am. Vet. Med. Assoc. 2013, 243, 1270-1288.

34. Keen, J.E.; Wittum, T.E.; Dunn, J.R.; Bono, J.L.; Durso, L.M. Shiga-toxigenic Escherichia coli O157 in agricultural fair livestock, United States. Emerg. Infect. Dis. 2006, 12, 780-786. [CrossRef] [PubMed] 
35. Goode, B.; O’Reilly, C.; Dunn, J.; Fullerton, K.; Smith, S.; Ghneim, G.; Keen, J.; Durso, L.; Davies, M.; Montgomery, S. Outbreak of Escherichia coli O157: H7 infections after petting zoo visits, North Carolina state fair, October-November 2004. Arch. Pediatr. Adolesc. Med. 2009, 163, 42-48. [CrossRef] [PubMed]

36. Varma, J.K.; Greene, K.D.; Reller, M.E.; DeLong, S.M.; Trottier, J.; Nowicki, S.F.; DiOrio, M.; Koch, E.M.; Bannerman, T.L.; York, S.T.; et al. An outbreak of Escherichia coli O157 infection following exposure to a contaminated building. Jama 2003, 290, 2709-2712. [CrossRef] [PubMed]

37. Steinmuller, N.; Demma, L.; Bender, J.B.; Eidson, M.; Angulo, F.J. Outbreaks of enteric disease associated with animal contact: Not just a foodborne problem anymore. Clin. Infect. Dis. 2006, 43, 1596-1602. [CrossRef] [PubMed]

38. Karmali, M.A.; Gannon, V.; Sargeant, J.M. Verocytotoxin-producing Escherichia coli (VTEC). Vet. Microbiol. 2010, 140, 360-370. [CrossRef] [PubMed]

39. Chandran, A.; Mazumder, A. Prevalence of diarrhea-associated virulence genes and genetic diversity in Escherichia coli isolates from fecal material of various animal hosts. Appl. Environ. Microbiol. 2013, 79, 7371-7380. [CrossRef] [PubMed]

40. Rostagno, M.H. Can stress in farm animals increase food safety risk? Foodborne Pathog. Dis. 2009, 6, 767-776. [CrossRef] [PubMed]

41. Besser, T.E.; Goldoft, M.; Pritchett, L.C.; Khakhria, R.; Hancock, D.D.; Rice, D.H.; Gay, J.M.; Johnson, W.; Gay, C.C. Multiresistant Salmonella Typhimurium DT104 infections of humans and domestic animals in the pacific northwest of the United States. Epidemiol. Infect. 2000, 124, 193-200. [CrossRef]

42. Sorensen, O.; Van Donkersgoed, J.; McFall, M.; Manninen, K.; Gensler, G.; Ollis, G. Salmonella spp. Shedding by Alberta beef cattle and the detection of Salmonella spp. in ground beef. J. Food Prot. 2002, 65, 484-491. [CrossRef] [PubMed]

43. Liljebjelke, K.A.; Hofacre, C.L.; Liu, T.; White, D.G.; Ayers, S.; Young, S.; Maurer, J.J. Vertical and horizontal transmission of Salmonella within integrated broiler production system. Foodborne Pathog. Dis. 2005, 2, 90-102. [CrossRef] [PubMed]

44. Dressler, A.E.; Scheibel, R.P.; Wardyn, S.; Harper, A.L.; Hanson, B.M.; Kroeger, J.S.; Diekema, D.J.; Bender, J.B.; Gray, G.C.; Smith, T.C. Prevalence, antibiotic resistance and molecular characterisation of Staphylococcus aureus in pigs at agricultural fairs in the USA. Vet. Rec. 2012, 170, 495. [CrossRef] [PubMed]

45. Khanna, T.; Friendship, R.; Dewey, C.; Weese, J.S. Methicillin resistant Staphylococcus aureus colonization in pigs and pig farmers. Vet. Microbiol. 2008, 128, 298-303. [CrossRef] [PubMed]

46. Oporto, B.; Esteban, J.I.; Aduriz, G.; Juste, R.A.; Hurtado, A. Prevalence and strain diversity of thermophilic campylobacters in cattle, sheep and swine farms. J. Appl. Microbiol. 2007, 103, 977-984. [CrossRef] [PubMed]

47. Moriarty, E.M.; Downing, M.; Bellamy, J.; Gilpin, B.J. Concentrations of faecal coliforms, Escherichia coli, Enterococci and Campylobacter spp. In equine faeces. N. Z. Vet. J. 2015, 63, 104-109. [CrossRef] [PubMed]

48. Pintar, K.D.; Christidis, T.; Thomas, M.K.; Anderson, M.; Nesbitt, A.; Keithlin, J.; Marshall, B.; Pollari, F. A systematic review and meta-analysis of the Campylobacter spp. prevalence and concentration in household pets and petting zoo animals for use in exposure assessments. PloS ONE 2015, 10, e0144976. [CrossRef] [PubMed]

49. Ryan, U.; Fayer, R.; Xiao, L. Cryptosporidium species in humans and animals: Current understanding and research needs. Parasitology 2014, 141, 1667-1685. [CrossRef] [PubMed]

50. Gow, S.P.; Waldner, C.L.; Harel, J.; Boerlin, P. Associations between antimicrobial resistance genes in fecal generic Escherichia coli isolates from cow-calf herds in western Canada. Appl. Environ. Microbiol. 2008, 74, 3658-3666. [CrossRef] [PubMed]

51. Scott, L.; Menzies, P.; Reid-Smith, R.J.; Avery, B.P.; McEwen, S.A.; Moon, C.S.; Berke, O. Antimicrobial resistance in fecal generic Escherichia coli and Salmonella spp. obtained from Ontario sheep flocks and associations between antimicrobial use and resistance. Can. J. Vet. Res. 2012, 76, 109-119. [PubMed]

52. Aarestrup, F.M.; Oliver Duran, C.; Burch, D.G. Antimicrobial resistance in swine production. Anim. Health Res. Rev. 2008, 9, 135-148. [CrossRef] [PubMed]

53. Benedict, K.M.; Gow, S.P.; McAllister, T.A.; Booker, C.W.; Hannon, S.J.; Checkley, S.L.; Noyes, N.R.; Morley, P.S. Antimicrobial resistance in Escherichia coli recovered from feedlot cattle and associations with antimicrobial use. PloS ONE 2015, 10, e0143995. [CrossRef] [PubMed] 
54. Sayah, R.S.; Kaneene, J.B.; Johnson, Y.; Miller, R. Patterns of antimicrobial resistance observed in Escherichia coli isolates obtained from domestic- and wild-animal fecal samples, human septage, and surface water. Appl. Environ. Microbiol. 2005, 71, 1394-1404. [CrossRef] [PubMed]

55. Chaudhuri, R.R.; Henderson, I.R. The evolution of the Escherichia coli phylogeny. Infect. Genet. Evol. 2012, 12, 214-226. [CrossRef] [PubMed]

56. Carlos, C.; Pires, M.M.; Stoppe, N.C.; Hachich, E.M.; Sato, M.I.; Gomes, T.A.; Amaral, L.A.; Ottoboni, L.M. Escherichia coli phylogenetic group determination and its application in the identification of the major animal source of fecal contamination. BMC Microbiol. 2010, 10, 161-171. [CrossRef] [PubMed]

(C) 2018 by the authors. Licensee MDPI, Basel, Switzerland. This article is an open access article distributed under the terms and conditions of the Creative Commons Attribution (CC BY) license (http://creativecommons.org/licenses/by/4.0/). 